\title{
Synthesis, Docking Studies and Anticancer Activity of New Substituted Pyrimidine and Triazolopyrimidine Glycosides
}

\author{
Wael A. El-Sayed ${ }^{1 *}$, Ashraf M. Mohamed ${ }^{2,3^{*}}$, Hemat S. Khalaf ${ }^{1}$, Dina S. EL-Kady ${ }^{4}$, May Al-Manawaty ${ }^{5}$ \\ ${ }^{1}$ Photochemistry Department, National Research Centre, Dokki, Giza, Egypt. \\ ${ }^{2}$ Chemistry Department, College of Science, Aljouf University, Sakaka, Al-Jouf, Kingdom of Saudi Arabia. \\ ${ }^{3}$ Applied Organic Chemistry Department, National Research Centre, Dokki, Cairo, Egypt. \\ ${ }^{4}$ Hormone Department, National Research Centre, Dokki, Cairo, Egypt. \\ ${ }^{5}$ Department of Pharmaceutical Chemistry, National Research Centre, Dokki, Cairo, Egypt.
}

\section{ARTICLE INFO \\ Article history: \\ Received on: 25/05/2017 \\ Accepted on: 13/07/2017 \\ Available online: 30/09/2017 \\ Key words: \\ Pyrimidine, \\ triazolopyrimidine, \\ glycosides, Enzyme Docking, \\ anticancer activity.}

\begin{abstract}
New substituted pyrimidine and triazolopyrimidine derivatives were synthesized. The $N^{3}$-glycosides of both heterocyclic systems and acyclic oxygenated alkyl derivatives were also prepared. The anticancer activity against human prostatic adenocarcinoma (PC3), human colorectal carcinoma (HCT116) and human breast adenocarcinoma (MCF7) cell lines in addition to their effect on human normal retinal pigmented epithelial cell line (RPE1) was studied. Furthermore, the docking studies revealed good binding affinities for compounds 7, 8 , 10 and 12. The results showed the effect of $N^{3}$-substitution in the pyrimidine ring on the activity of synthesized compounds.
\end{abstract}

\section{INTRODUCTION}

Although, recently, there have been developing advance in various therapeutic strategies, cytotoxic drugs remains the main backbone for cancer treatment (Butler et al., 2015). Drugs which affect DNA biosynthesis have received much attention and amongst them pyrimidine derivatives remain the most important (Cieplik, 1992; Pogorelcnik et al., 2015). Many pyrimidine incorporating compounds constitute an assortment of drugs with ability to hinder biosynthesis of pyrimidine nucleotides or act effectively as naturalist metabolites, thus interfering in substantial ellular processes, for example nucleic acids synthesis.

* Corresponding Authors

Email: waelshendy@gmail.com (El-Sayed W.A);

Email: ammewas1@gmail.com (Mohamed A.M.)
Pyrimidinone Pyrimidinones are present a class of such compounds attracting much interest because of their interesting pharmacological properties, as antitumor (Mohamed et al., 2013), antiviral (Al-Mohizea et al., 2012), anti-parkinsonism (Al-Harbi et al., 2013) and antimicrobial (Hossan et al., 2012) agents in addition to their application as building blocks for synthesizing new molecules (Wannberg et al., 2005). A variety of pyrimidine derivatives fused with other heterocycles has been found as anticancer agents used in clinics or in clinical trials (VanderWel et al., 2005; Toogood et al., 2005; Palmer et al., 2005; Malagu et al., 2009; Zhao et al., 2007; Hafez and El-Gazzar, 2009; Shawali et al., 2010). Derivatives of the $[1,2,4]$ triazolo[4,3-a]pyrimidine ring system have been revealed to possess antitumor activity (Zhao et al., 2007; Hafez and El-Gazzar, 2009; Shawali et al., 2010). On the other hand, a number of glycosides were investigated and have shown high anticancer activity (Aminin et al., 2016). 


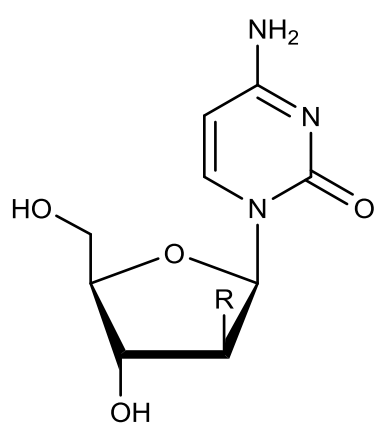

Cytarabine or Decitabine

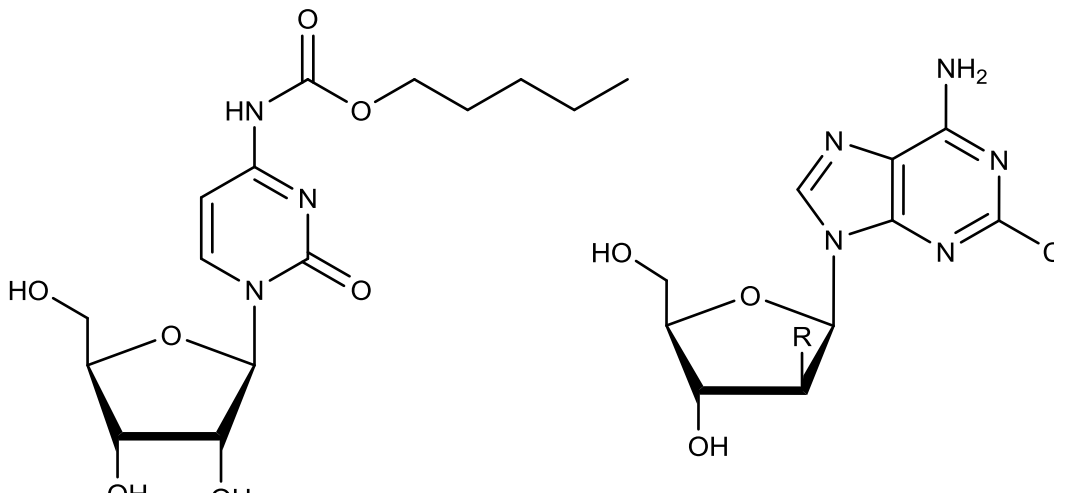

Capecitabine

Cladribine or Clofarabine

Fig. 1: Anticancer pyrimidine and fused pyrimidine nucleoside analogs.

Synthesized glycosides and their derived analogs (Zhao et al., 2008; Zhao and Li, 2007) revealed considerable inhibitory activity of DNA-topoisomerase II (TopoII) that controls DNA topology by transitory cleavage of the DNA double helix, which was affirmed as important molecular target of anticancer drugs (Champoux, 2001; Pommier et al., 2010). Moreover, studied biological assays found that exotic sugar part confers a number of glycosides with best conformation possessing high binding affinities via hydrogen bonding to the entry of the ATPase pocket (Gui et al., 2011; Shi et al., 2012). In previous docking studies, it has been shown that acetylated and deprotected sugar moieties play very important roles in their found significant inhibition activity (Zhao et al., 2012). Nucleoside analogs have been investigated as important drugs for the treatment of different cancer types. Compounds such as Cytarabine, Fludarabine, Cladribine, Gemcitabine, Clofarabine, Capecitabine, Floxuridine, Deoxycoformycin, Azacitidine and Decitabine (fig. 1) which belong to the nucleoside analogs class, currently approved antitumoral activity (Jordheim et al., 2013). Different nucleoside analogs incorporating substituents at the $C-5$ position in the heterocyclic base, especially in the 2-deoxyuridine type, were investigated to have interesting biological activities as antiviral and anticancer agents (Srinivasan et al., 20101; McGuian et al., 2000; Yoo et al., 2002; Hannah et al., 2000; Khan and Grinstaff, 1998). The above findings and our interest in glycosyl heterocycles synthesis with biological activity research field (Mohamed et al., 2015; El-Sayed et al, 2009; Amr et al., 2006; ElSayed et al., 2016) promoted us to design and synthesize new pyrimidine and triazolopyrimidine glycosides with acetylated or free hydroxyl sugar moieties and acyclic analogs bearing ether or terminal hydroxyl studying their anticancer activity against a number of cancer cells.

\section{MATERIALS AND METHODS}

\section{Instruments and reagents}

Melting points were measured using a Böetius PHMK (VebAnalytik Dresden) instrument. TLC was implemented using aluminum plates pre-coated with silica gel 60 or 60 F254 (Merck) and visualized using UV light (254 nm). Nuclear Magnetic Resonance of compounds was carried out on a Varian Gemini 300 and Bruker DRX 400 spectrometer at $25{ }^{\circ} \mathrm{C}$ with TMS as a reference and solvent shift $\left(\left(\mathrm{CD}_{3}\right)_{2} \mathrm{SO} \delta \mathrm{H} 2.50\right.$ and $\left.\delta \mathrm{C} 39.5\right)$. Coupling constants are expressed in $\mathrm{Hz}$ without sign. Mass spectrometry was performed using a Varian FINNIGAN MAT 212 machine. The Infra-red spectra were investigated (KBr) by means of a Jasco FT/IR-410 apparatus. Elemental analysis was measured using the Perkin Elmer 240 instrument. The starting hydrazinyl pyrimidine compound $\mathbf{1}$ was prepared as previously reported (Libermann and Rouaix, 1955).

\section{Synthesis \\ 2-(2-((5-Methylfuran-2-yl)methylene)hydrazinyl)-6- propylpyrimidin-4(3H)-one (2)}

A solution of 5-methylfurfural $(0.01 \mathrm{~mol})$ and 2 hydrazinyl-6-propylpyrimidin-4 $(3 H)$-one $(0.01 \mathrm{~mol})$ was heated at reflux temperature in absolute ethanol $(45 \mathrm{~mL})$ in presence of piperidine (two drops) for $4 \mathrm{~h}$, whereby, a precipitate was being formed. The precipitated product was collected, dried followed by crystallization from ethanol to afford the hydrazinyl derivative.

Yield 84\%; m.p. $240-241^{\circ} \mathrm{C}$; IR (KBr): $3220(\mathrm{NH}), 1664$ $\mathrm{cm}^{-1}(\mathrm{C}=\mathrm{O}) .{ }^{1} \mathrm{H}$ NMR (DMSO-d $)$ ) $\delta / \mathrm{ppm}: 0.89(\mathrm{t}, J=6.4 \mathrm{~Hz}, 3 \mathrm{H}$, $\left.\mathrm{CH}_{3}\right), 1.58-1.64\left(\mathrm{~m}, 2 \mathrm{H}, \mathrm{CH}_{2}\right), 2.33\left(\mathrm{~s}, 3 \mathrm{H}, \mathrm{CH}_{3}\right), 2.38(\mathrm{t}, J=6.4$ $\left.\mathrm{Hz}, 2 \mathrm{H}, \mathrm{CH}_{2}\right), 6.24$ (s, 1H, pyrimidine-H), 6.34 (d, J=7.8 Hz, 1H, ArH), 6.97 (d, J = 7.8 Hz, 1H, ArH), 7.98 (s, 1H, N=CH), 10.80 (1H, s, NH), 11.02 (s, 1H, NH); ${ }^{13} \mathrm{C}$ NMR (DMSO-d 6 ) $\delta / p p m:$ $13.9\left(\mathrm{CH}_{3}\right), 21.1\left(\mathrm{CH}_{2}\right), 39.4\left(\mathrm{CH}_{2}\right), 101.1$ (pyrimidine $\left.\mathrm{C}-5\right), 109.1$ (Ar-C), 109.6 (Ar-C), 114.3-156.5 (Ar-C and $\mathrm{C}=\mathrm{N}), 172.5(\mathrm{C}=\mathrm{O})$. MS [m/z]: $259\left(\mathrm{M}^{+}-1,10 \%\right)$. Analysis calcd. for $\mathrm{C}_{13} \mathrm{H}_{16} \mathrm{~N}_{4} \mathrm{O}_{2}: \mathrm{C}$, 59.99; H, 6.20; N, 21.52. Found: C, 60.27; H, 6.35; N, 21.18.

\section{3-(2-(2-Hydroxyethoxy)ethyl) -2- (2-((5-methylfuran-2-yl) methylene)hydrazine-yl)-6-propylpyrimidin-4(3H)-one (3)}

To a solution of substituted pyrimidine $2(0.01 \mathrm{~mol})$ in DMF $(10 \mathrm{~mL})$ in crushed ice bath, was provided a cold stirred 
suspension of sodium hydride $(0.02 \mathrm{~mol})$ in DMF $(5 \mathrm{~mL})$ portion wise over a period of 10 minutes at $0{ }^{\circ} \mathrm{C}$, then stirring was continued for 1 hour at room temperature. 2-(2-Chloroethoxy) ethanol $(0.01 \mathrm{~mol})$ was added and the reaction mixture was further stirred for $6 \mathrm{~h}$ at room temperature. The solid material was filtered off and washed with DMF ( $5 \mathrm{~mL})$. The washings and filtrate were combined and the remaining residue, after removal of solvent, was processed in column chromatography (pet. ether/EtOAc; 3:1) giving 3

Yield 77\%; m.p. $258-259^{\circ} \mathrm{C}$; IR (KBr): $3315(\mathrm{OH}), 3225$ $(\mathrm{NH})$ and $1655 \mathrm{~cm}^{-1}(\mathrm{CO}) .{ }^{1} \mathrm{H} \mathrm{NMR}\left(\mathrm{CDCl}_{3}\right) \delta / \mathrm{ppm}: 0.87(\mathrm{t}, J=6.2$ $\left.\mathrm{Hz}, 3 \mathrm{H}, \mathrm{CH}_{3}\right), 1.36-1.41\left(\mathrm{~m}, 2 \mathrm{H}, \mathrm{CH}_{2}\right), 2.25(\mathrm{t}, J=6.4 \mathrm{~Hz}, 2 \mathrm{H}$, $\left.\mathrm{CH}_{2}\right), 2.41\left(\mathrm{~s}, 3 \mathrm{H}, \mathrm{CH}_{3}\right), 3.50\left(\mathrm{t}, J=6.2 \mathrm{~Hz}, 2 \mathrm{H}, \mathrm{CH}_{2}\right), 3.59(\mathrm{t}, J=$ $\left.6.2 \mathrm{~Hz}, 2 \mathrm{H}, \mathrm{CH}_{2}\right), 3.83-3.87\left(\mathrm{~m}, 2 \mathrm{H}, \mathrm{CH}_{2}\right), 4.35$ (t, $J=6.4 \mathrm{~Hz}, 2 \mathrm{H}$, $\left.\mathrm{CH}_{2}\right), 5.35(\mathrm{~m}, 1 \mathrm{H}, \mathrm{OH}), 6.27(\mathrm{~s}, 1 \mathrm{H}$, pyrimidine- $\mathrm{H}), 6.35(\mathrm{~d}, J=$ $7.8 \mathrm{~Hz}, 1 \mathrm{H}, \mathrm{ArH}), 6.90$ (d, J = 7.8 Hz, 1H, ArH), 7.99 (s, 1H, $\mathrm{N}=\mathrm{CH}), 10.12$ (brs, 1H, NH). MS [m/z]: $349\left(\mathrm{M}^{+}+1,14 \%\right)$. Analysis calcd. for $\mathrm{C}_{17} \mathrm{H}_{24} \mathrm{~N}_{4} \mathrm{O}_{4}: \mathrm{C}, 58.61 ; \mathrm{H}, 6.94 ; \mathrm{N}, 16.08$. Found: C, 58.48; H, 6.75; N, 16.89\%.

\section{3-(2,2-Dimethoxyethyl)-2- (2- ((5-methylfuran-2-yl) methylene)} hydrazinyl)-6-propylpyrimidin-4(3H)-one (4)

A suspension of sodium hydride $(0.03 \mathrm{~mol})$ in DMF (5 $\mathrm{mL})$ was dropped into a solution of pyrimidine $2(0.01 \mathrm{~mol})$ in DMF $(10 \mathrm{~mL})$ portion wise in an ice bath over a period of 15 minutes at $0{ }^{\circ} \mathrm{C}$, with persistent stirring for 1 hour at room temperature. 2-Chloro-1,1-dimethoxyethane was added and the reaction mixture was stirred for $10 \mathrm{~h}$ (TLC) at room temperature. Ice-cold water was added and the resulting emulsion was further stirred in crushed ice for about 2 h. Filtration and dryness of the formed solid followed by recrystallizing in methanol produced the dimethoxyethyl derivative 4.

Yield 75\%; m.p. 221-222 ${ }^{\circ} \mathrm{C}$; IR (KBr): $3223(\mathrm{NH})$ and $1660 \mathrm{~cm}^{-1}(\mathrm{C}=\mathrm{O}) .{ }^{1} \mathrm{H}$ NMR $\left(\mathrm{CDCl}_{3}\right) \delta / \mathrm{ppm}: 0.88(\mathrm{t}, J=5.8,3 \mathrm{H}$, $\left.\mathrm{CH}_{3}\right), 1.57\left(\mathrm{~m}, 2 \mathrm{H}, \mathrm{CH}_{2}\right), 2.23\left(\mathrm{t}, J=5.8,2 \mathrm{H}, \mathrm{CH}_{2}\right), 2.38(\mathrm{~s}, 3 \mathrm{H}$, $\left.\mathrm{CH}_{3}\right), 3.43\left(\mathrm{~s}, 6 \mathrm{H}, 2 \mathrm{OCH}_{3}\right), 4.01\left(\mathrm{~d}, J=5.8,2 \mathrm{H}, \mathrm{CH}_{2}\right), 5.48(\mathrm{t}, \mathrm{J}=$ $6.4 \mathrm{~Hz}, 1 \mathrm{H}, \mathrm{O}-\mathrm{CH}-\mathrm{O}), 6.23$ (s, 1H, pyrimidine-H), 6.52 (d, $J=7.8$ $\mathrm{Hz}, 1 \mathrm{H}, \mathrm{ArH}), 6.89$ (d, J = 7.8 Hz, 1H, Ar-H), 8.07 (s, 1H, N=CH), 10.09(s, 1H, NH). MS [m/z]: $348\left(\mathrm{M}^{+}, 11 \%\right)$. Analysis calcd. For $\mathrm{C}_{17} \mathrm{H}_{24} \mathrm{~N}_{4} \mathrm{O}_{4}$ : C, 58.61; H, 6.94; N, 16.08. Found: C, 58.47; H, $6.77 ; \mathrm{N}, 16.21 \%$.

\section{Synthesis of acetylated $N$-glycosides 6 and 7}

A solution of compound $2(0.01 \mathrm{~mol})$ in DMF $(15 \mathrm{~mL})$ was added portion wise to a stirred suspension of sodium hydride $(0.015 \mathrm{~mol})$ at $0^{\circ} \mathrm{C}$ then stirring was continued for $1 \mathrm{~h}$ at room temperature. Acetylated bromo-sugar 5a, b $(0.01 \mathrm{~mol})$ dissolved in DMF $(10 \mathrm{~mL})$ was added slowly to the mixture then continual stirring was performed for 6-8 h (TLC eluent: pet. ether/hexane, 3:1). Ice cold water was added with vigorous stirring for 30 minutes and the resulting precipitate was filtered, dried and crystallized from ethanol.
2-(Acetoxymethyl)- 6-(2-(2-((5-methylfuran-2-yl)methylene) hydrazinyl)-6-oxo-4-propylpyrimidin-1(6H)-yl)tetrahydro-2Hpyran-3,4,5-triyl triacetate (6)

Yield 74\%; m.p. 158-159 ${ }^{\circ} \mathrm{C}$; IR (KBr): $3296(\mathrm{NH}), 1753$ $(\mathrm{C}=\mathrm{O}), 1666 \mathrm{~cm}^{-1}(\mathrm{C}=\mathrm{O}) .{ }^{1} \mathrm{H}$ NMR $\left(\mathrm{CDCl}_{3}, 300 \mathrm{MHz}\right) \delta / \mathrm{ppm}: 0.89$ $\left(\mathrm{t}, J=6.2 \mathrm{~Hz}, 3 \mathrm{H}, \mathrm{CH}_{3}\right), 1.58\left(\mathrm{~m}, 2 \mathrm{H}, \mathrm{CH}_{2}\right), 1.97,1.99,2.02,2.04$ $\left(4 \mathrm{~s}, 12 \mathrm{H}, 4 \mathrm{CH}_{3}\right), 2.34\left(\mathrm{t}, J=6.2 \mathrm{~Hz}, 2 \mathrm{H}, \mathrm{CH}_{2}\right), 2.41\left(\mathrm{~s}, 3 \mathrm{H}, \mathrm{CH}_{3}\right)$, $4.03\left(\mathrm{dd}, J=10.2 \mathrm{~Hz}, J=3.5 \mathrm{~Hz}, 1 \mathrm{H}, \mathrm{H}-5^{\prime}\right), 4.12(\mathrm{dd}, J=3.8,10.2$ $\left.\mathrm{Hz}, 1 \mathrm{H}, \mathrm{H}-6^{\prime \prime}\right), 4.15$ (dd, $\left.J=11.3,3.8 \mathrm{~Hz}, 1 \mathrm{H}, \mathrm{H}-6^{\prime}\right), 5.22$ (t, $J=$ $\left.3.2 \mathrm{~Hz}, 1 \mathrm{H}, \mathrm{H}-4^{\prime}\right), 5.26\left(\mathrm{dd}, J=6.6,3.2 \mathrm{~Hz}, 1 \mathrm{H}, \mathrm{H}-3^{\prime}\right), 5.32(\mathrm{t}, J=$ $\left.6.6 \mathrm{~Hz}, 1 \mathrm{H}, \mathrm{H}-2^{\prime}\right), 5.98$ (d, $\left.J=10.2 \mathrm{~Hz}, 1 \mathrm{H}, \mathrm{H}-1^{\prime}\right), 6.27$ (s, 1H, pyrimidine-H), 6.29 (d, $J=7.8 \mathrm{~Hz}, 1 \mathrm{H}, \mathrm{Ar}-\mathrm{H}), 6.92$ (d, $J=7.8 \mathrm{~Hz}$, $1 \mathrm{H}, \mathrm{Ar}-\mathrm{H}), 7.98$ (s, 1H, N=CH), 9.45 (s, 1H, NH); ${ }^{13} \mathrm{C}$ NMR $\left(\right.$ DMSO-d $\left._{6}\right) \delta /$ ppm: $13.9\left(\mathrm{CH}_{3}\right), 20.7\left(\mathrm{CH}_{2}\right), 20.9,21.0,21.1,21.5$ $\left(4 \mathrm{CH}_{3}\right), 38.2\left(\mathrm{CH}_{2}\right), 62.3(\mathrm{C}-6), 68.5(\mathrm{C}-4), 68.9(\mathrm{C}-3), 71.2(\mathrm{C}-2)$, 78.3 (C-5), 93.3 (C-1), 101.9 (pyrimidine C-5), 109.1 (Ar-C), 109.6 (Ar-C), 114.4-156.6 (Ar-C and $\mathrm{C}=\mathrm{N}), 169.5$, 169.8, 170.0, 170.1, $172.5(5 \mathrm{C}=\mathrm{O})$. Analysis calcd. for $\mathrm{C}_{27} \mathrm{H}_{34} \mathrm{~N}_{4} \mathrm{O}_{11}: \mathrm{C}, 54.91$; H, 5.80; N, 9.49. Found: C, 54.70; H, 5.65; N, 9.68\%.

\section{2-(2-(2-((5-Methylfuran-2-yl)methylene)hydrazinyl)-6-oxo-4- propylpyrimidin-1 $(6 H)$-yl)tetrahydro-2H-pyran-3,4,5-triyl triacetate (7)}

Yield 76\%; m.p. $165-166^{\circ} \mathrm{C}$; IR (KBr): $3284(\mathrm{NH}), 1752$ $(\mathrm{C}=\mathrm{O}), 1668 \mathrm{~cm}^{-1}(\mathrm{C}=\mathrm{O}) .{ }^{1} \mathrm{H} \mathrm{NMR}\left(\mathrm{DMSO}_{-} \mathrm{d}_{6}\right) \delta / \mathrm{ppm}: 0.90(\mathrm{t}, J=$ $\left.6.2 \mathrm{~Hz}, 3 \mathrm{H}, \mathrm{CH}_{3}\right), 1.57\left(\mathrm{~m}, 2 \mathrm{H}, \mathrm{CH}_{2}\right), 1.99,2.02,2.04(3 \mathrm{~s}, 9 \mathrm{H}, 3$ $\left.\mathrm{CH}_{3}\right), 2.33$ (t, $\left.J=6.2 \mathrm{~Hz}, 2 \mathrm{H}, \mathrm{CH}_{2}\right), 2.39$ (s, 3H, $\left.\mathrm{CH}_{3}\right), 4.15$ (dd, $J$ $\left.=3.8,10.2 \mathrm{~Hz}, 1 \mathrm{H}, \mathrm{H}-5^{\prime \prime}\right), 4.19$ (dd, $\left.J=11.3,3.8 \mathrm{~Hz}, 1 \mathrm{H}, \mathrm{H}-5^{\prime}\right)$, $5.23\left(\mathrm{t}, J=3.2 \mathrm{~Hz}, 1 \mathrm{H}, \mathrm{H}-4^{\prime}\right), 5.27\left(\mathrm{dd}, J=6.6,3.2 \mathrm{~Hz}, 1 \mathrm{H}, \mathrm{H}-3^{\prime}\right)$, $5.33\left(\mathrm{t}, J=6.6 \mathrm{~Hz}, 1 \mathrm{H}, \mathrm{H}-2^{\prime}\right), 5.97$ (d, J = 9.4 Hz, 1H, H-1'), 6.26 (s, 1H, pyrimidine-H), $6.30(\mathrm{~d}, J=7.8 \mathrm{~Hz}, 1 \mathrm{H}, \mathrm{Ar}-\mathrm{H}), 6.90(\mathrm{~d}, J=$ $7.8 \mathrm{~Hz}, 1 \mathrm{H}, \mathrm{Ar}-\mathrm{H}), 7.90(\mathrm{~s}, 1 \mathrm{H}, \mathrm{N}=\mathrm{CH}), 9.08(\mathrm{~s}, 1 \mathrm{H}, \mathrm{NH}) .{ }^{13} \mathrm{C}$ NMR (DMSO-d $\left.{ }_{6}\right) \delta / p p m: ~ 14.8\left(\mathrm{CH}_{3}\right), 18.7\left(\mathrm{CH}_{2}\right), 20.1,20.7,20.8$ $\left(4 \mathrm{CH}_{3}\right), 39.3\left(\mathrm{CH}_{2}\right), 62.7(\mathrm{C}-5), 67.1(\mathrm{C}-4), 68.2(\mathrm{C}-3), 73.7(\mathrm{C}-2)$, 91.4 (C-1), 101.3 (pyrimidine C-5), 107.8 (Ar-C), 108.7 (Ar-C), 123.3-154.5 (Ar-C and $\mathrm{C}=\mathrm{N}), 170.8,171.2,171.6,171.9(4 \mathrm{C}=\mathrm{O})$. Analysis calcd. for $\mathrm{C}_{24} \mathrm{H}_{30} \mathrm{~N}_{4} \mathrm{O}_{9}: \mathrm{C}, 55.59 ; \mathrm{H}, 5.83 ; \mathrm{N}, 10.81$. Found: C, 55.35; H, 5.95; N, 10.71\%.

\section{Synthesis of deacetylated $N$-glycosides 8 and 9}

A solution of the acetylated glycoside 6 and $7(0.5 \mathrm{~g})$ in saturated methanolic ammonia $(20 \mathrm{~mL})$ was stirred at room temperature for $5 \mathrm{~h}$. After completion of the deacetylation process (TLC: petroleum ether/hexane, 2:1), the liquid was vaporized by means of rotatory evaporator and the remnant was triturated with diethyl ether $(25 \mathrm{~mL})$ leading to a solid which was filtered, dried and crystallized from ethanol.

2-(2-((5-Methylfuran-2-yl)methylene)hydrazinyl)-6-propyl-3(3,4,5-trihydroxy-6-(hydroxymethyl)tetrahydro-2H-pyran-2yl)pyrimidin-4(3H)-one $(8)$

Yield 78\%; m.p. 205-206 ${ }^{\circ}$; IR (KBr): 3490-3446 (OH), $3288 \mathrm{~cm}^{-1}(\mathrm{NH}), 1675(\mathrm{C}=\mathrm{O}) .{ }^{1} \mathrm{H}$ NMR (DMSO-d $\left.{ }_{6}\right) \delta / \mathrm{ppm}: 0.91(\mathrm{t}$, $\left.J=6.2 \mathrm{~Hz}, 3 \mathrm{H}, \mathrm{CH}_{3}\right), 1.58-1.61\left(\mathrm{~m}, 2 \mathrm{H}, \mathrm{CH}_{2}\right), 2.32(\mathrm{t}, J=6.2 \mathrm{~Hz}$, 
2H, $\mathrm{CH}_{2}$ ), 2.38 (s, 3H, $\mathrm{CH}_{3}$ ), 3.44-3.51 (m, 2H, H-6',6"), 3.60-3.66 (m, $\left.1 \mathrm{H}, \mathrm{H}-5^{\prime}\right), 3.79-3.86$ (m, 2H, H-4', $\left.3^{\prime}\right)$, 4.12-4.15 (m, 1H, OH), 4.65-4.77 (m, 2H, OH and H-2'), 4.86-4.94 (m, 2H, 2 OH), 5.80 $\left(\mathrm{d}, 1 \mathrm{H}, J=8.2 \mathrm{~Hz}, \mathrm{H}-1^{\prime}\right), 6.24(\mathrm{~s}, 1 \mathrm{H}$, pyrimidine-H), $6.32(\mathrm{~d}, J=$ $7.8 \mathrm{~Hz}, 1 \mathrm{H}, \mathrm{Ar}-\mathrm{H}), 6.91$ (d, $J=7.8 \mathrm{~Hz}, 1 \mathrm{H}, \mathrm{Ar}-\mathrm{H}), 7.92$ (s, 1H, $\mathrm{N}=\mathrm{CH}), 9.11(\mathrm{~s}, 1 \mathrm{H}, \mathrm{NH})$. Analysis calcd. for $\mathrm{C}_{19} \mathrm{H}_{26} \mathrm{~N}_{4} \mathrm{O}_{7}: \mathrm{C}$, 54.02; H, 6.20; N, 13.26. Found: C, 44.91; H, 6.14; N, $13.05 \%$.

\section{2-(2-((5-Methylfuran-2-yl)methylene)hydrazinyl)-6-propyl-3-} (3,4,5-trihydroxytetrahydro-2H-pyran-2-yl)pyrimidin-4(3H)one (9)

Yield 73\%; m.p. 210-211 ${ }^{\circ} \mathrm{C}$; IR (KBr): 3490-3446 (OH), $3288 \mathrm{~cm}^{-1}(\mathrm{NH}), 1670(\mathrm{C}=\mathrm{O}) .{ }^{1} \mathrm{H}$ NMR $\left(\mathrm{DMSO}_{-} \mathrm{d}_{6}\right) \delta / \mathrm{ppm}: 0.90(\mathrm{t}$, $\left.J=6.2 \mathrm{~Hz}, 3 \mathrm{H}, \mathrm{CH}_{3}\right), 1.57-1.61\left(\mathrm{~m}, 2 \mathrm{H}, \mathrm{CH}_{2}\right), 2.31(\mathrm{t}, J=6.2 \mathrm{~Hz}$, $2 \mathrm{H}, \mathrm{CH}_{2}$ ), 2.37 (s, 3H, $\mathrm{CH}_{3}$ ), 3.59-3.71 (m, 2H, H-5',5"), 3.80-3.88 (m, 2H, H-4', $\left.3^{\prime}\right), 4.21$ (m, 1H, OH), 4.40-4.46 (m, 1H, OH), 4.534.58 (m, 1H, H-2'), 4.69-4.75 (m, 1H, OH), 5.79 (d, 1H, J=8.2 Hz, H-1'), 6.22 (s, 1H, pyrimidine $-\mathrm{H}), 6.32$ (d, $J=7.8 \mathrm{~Hz}, 1 \mathrm{H}, \mathrm{Ar}-\mathrm{H})$, 6.90 (d, J = 7.8 Hz, 1H, Ar-H), 7.91 (s, 1H, N=CH), 9.08 (s, 1H, $\mathrm{NH})$. Analysis calcd. for $\mathrm{C}_{18} \mathrm{H}_{24} \mathrm{~N}_{4} \mathrm{O}_{6}: \mathrm{C}, 55.09 ; \mathrm{H}, 6.16 ; \mathrm{N}, 14.28$. Found: C, 54.81; H, 5.94; N, 14.20\%.

\section{3-(5-Methylfuran-2-yl)-5-propyl-[1,2,4]triazolo[4,3- a]pyrimidin-7(8H)-one (10)}

To a mixture of the hydrazone derivative $2(0.003 \mathrm{~mol})$ and two molar equivalents of sodium acetate in dry methanol(50 $\mathrm{mL})$, was provided a solution of bromine $(0.16 \mathrm{~mL}, 0.003 \mathrm{~mol})$ in anhydrous methanol $(5 \mathrm{~mL})$ over a period of 10 minutes at $0{ }^{\circ} \mathrm{C}$. The mixture was then stirred for 12 hours at room temperature. The solvent was then removed on a rotary evaporator then the remaining gum was triturated with a dry diethyl ether/pet. ether $(1: 1,25 \mathrm{~mL})$ with shaking to form a solid material which was collected and dried affording triazolopyrimidine 10 .

Yield 69\%; m.p. $223-224^{\circ} \mathrm{C}$; IR (KBr): $3220(\mathrm{NH})$, $1668 \mathrm{~cm}^{-1}(\mathrm{C}=\mathrm{O}) .{ }^{1} \mathrm{H}$ NMR (DMSO-d $\left.{ }_{6}\right) \delta / \mathrm{ppm}: 0.94(\mathrm{t}, J=6.4 \mathrm{~Hz}$, $\left.3 \mathrm{H}, \mathrm{CH}_{3}\right), 1.66\left(\mathrm{~m}, 2 \mathrm{H}, \mathrm{CH}_{2}\right), 2.34\left(\mathrm{~s}, 3 \mathrm{H}, \mathrm{CH}_{3}\right), 2.39$ (t, $J=6.4$ $\left.\mathrm{Hz}, 2 \mathrm{H}, \mathrm{CH}_{2}\right), 6.29$ (s, 1H, pyrimidine-H), 6.95 (d, J=7.8 Hz, 1H, ArH), 7.95 (d, $J=7.8 \mathrm{~Hz}, 1 \mathrm{H}, \mathrm{ArH}), 10.80(1 \mathrm{H}, \mathrm{s}, \mathrm{NH}) .{ }^{13} \mathrm{C} \mathrm{NMR}$

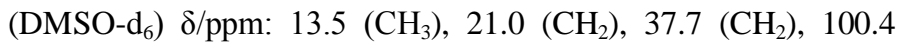
(pyrimidine C-5), 108.6 (Ar-C), 113.7 (Ar-C), 134.8-154.1 (Ar-C and $2 \mathrm{C}=\mathrm{N}), 162.0(\mathrm{C}=\mathrm{O})$. MS [m/z]: $258\left(\mathrm{M}^{+}, 7 \%\right)$. Analysis Calcd. for $\mathrm{C}_{13} \mathrm{H}_{14} \mathrm{~N}_{4} \mathrm{O}_{2}: \mathrm{C}, 60.45 ; \mathrm{H}, 5.46 ; \mathrm{N}, 21.69$. Found: $\mathrm{C}$, $60.24 ; \mathrm{H}, 5.31 ; \mathrm{N}, 21.82$.

\section{Synthesis of the $N$-substituted triazolopyrimidine derivatives 11 and 12}

Method A: To a well stirred suspension of sodium hydride $(0.05 \mathrm{~mol})$ in dry DMF $(15 \mathrm{~mL})$ at $0^{\circ} \mathrm{C}$, a solution of the triazolopyrimidine $\mathbf{1 0}(0.01 \mathrm{~mol})$ in DMF $(20 \mathrm{~mL})$ was added portion-wise through 15 minutes while the mixture was preserved in cooling ice. The ice was removed and the reaction contents were stirred for 1 hour. 2-(2-Chloroethoxy) ethanol or chloroacetaldehyde dimethyl acetal $(0.01 \mathrm{~mol})$ was inserted then the mixture was stirred for $7-10 \mathrm{~h}$ at $70{ }^{\circ} \mathrm{C}$. After completion of the reaction (TLC: petroleum ether/ethyl acetate, 3:1), ice-cold water was added and the resulting gum substance was further stirred with crushed ice for about $1 \mathrm{~h}$. Filtration of the precipitate and recrystallization from ethanol resulted in the acyclic oxygenated compounds $\mathbf{1 1}$ or $\mathbf{1 2}$, respectively.

\section{Method B (for compound 12)}

Anhydrous sodium acetate $(0.01 \mathrm{~mol})$ was added to a solution of compound $4(0.01 \mathrm{~mol})$ in acetic acid $(20 \mathrm{~mL})$ and the resulting mixture was heated till dissolution of the solid material. The reaction flask was cooled by means of ice-water bath then acetic acid $(5 \mathrm{~mL})$ containing bromine $(0.015 \mathrm{~mol})$ was slowly added drop-wise during 10-15 minutes. The afforded oily material was shacked continuously at room temperature for 30 minutes followed by heating to $100{ }^{\circ} \mathrm{C}$ for 1 hour. The mixture was cooled then poured onto crushed ice and the precipitated triazolopyrimidine was collected by filtration, and crystallized from cold methanol.

8-(2-(2-Hydroxyethoxy)ethyl)-3-(5-methylfuran-2-yl)5-propyl-[1,2,4]triazolo[4,3-a]pyrimidin-7(8H)-one (11)

Yield 77\%; m.p. $238-239{ }^{\circ} \mathrm{C}$; IR (KBr): $3315 \mathrm{~cm}^{-1}(\mathrm{OH})$, $1659 \mathrm{~cm}^{-1}(\mathrm{C}=\mathrm{O}) .{ }^{1} \mathrm{H}$ NMR (DMSO-d $\left.{ }_{6}\right) \delta / \mathrm{ppm}: 0.92(\mathrm{t}, J=4.8 \mathrm{~Hz}$, $\left.3 \mathrm{H}, \mathrm{CH}_{3}\right), 1.35\left(\mathrm{~m}, 2 \mathrm{H}, \mathrm{CH}_{2}\right), 1.99\left(\mathrm{t}, J=5.2 \mathrm{~Hz}, 2 \mathrm{H}, \mathrm{CH}_{2}\right), 2.42$ $\left(\mathrm{s}, 3 \mathrm{H}, \mathrm{CH}_{3}\right), 3.50\left(\mathrm{t}, J=5.4 \mathrm{~Hz}, 2 \mathrm{H}, \mathrm{CH}_{2}\right), 3.65(\mathrm{t}, J=5.4 \mathrm{~Hz}, 2 \mathrm{H}$, $\left.\mathrm{CH}_{2}\right), 3.74-3.78\left(\mathrm{~m}, 2 \mathrm{H}, \mathrm{CH}_{2}\right), 3.94\left(\mathrm{t}, J=5.6 \mathrm{~Hz}, 2 \mathrm{H}, \mathrm{CH}_{2}\right), 4.80$ $(\mathrm{m}, 1 \mathrm{H}, \mathrm{OH}), 6.22(\mathrm{~s}, 1 \mathrm{H}$, pyrimidine-H), $6.40(\mathrm{~d}, 1 \mathrm{H}, \mathrm{ArH}), 7.10$ (d, $J=8.2 \mathrm{~Hz}, 1 \mathrm{H}, \mathrm{ArH}) . \mathrm{MS}[\mathrm{m} / \mathrm{z}]: 346\left(\mathrm{M}^{+}, 5 \%\right)$. Analysis calcd. For $\mathrm{C}_{17} \mathrm{H}_{22} \mathrm{~N}_{4} \mathrm{O}_{4}$ : C, 58.95; H, 6.40; N, 16.17. Found: C, 58.72; H, $6.29 ; \mathrm{N}, 16.05 \%$.

\section{8-(2,2-Dimethoxyethyl)-3-(5-methylfuran-2-yl)-5-propyl- $[1,2,4]$ triazolo[4,3-a]pyrimidin-7(8H)-one (12)}

Yield 79\%; m.p. $206-207^{\circ} \mathrm{C}$; IR (KBr): $1658 \mathrm{~cm}^{-1}(\mathrm{C}=\mathrm{O})$. ${ }^{1} \mathrm{H} \mathrm{NMR}\left(\mathrm{CDCl}_{3}\right) \delta / \mathrm{ppm}: 1.07\left(\mathrm{t}, J=5.8,3 \mathrm{H}, \mathrm{CH}_{3}\right), 1.70(\mathrm{~m}, 2 \mathrm{H}$, $\left.\mathrm{CH}_{2}\right), 2.02\left(\mathrm{t}, J=5.8,2 \mathrm{H}, \mathrm{CH}_{2}\right), 2.37\left(\mathrm{~s}, 3 \mathrm{H}, \mathrm{CH}_{3}\right), 3.48(\mathrm{~s}, 6 \mathrm{H}$, $\left.2 \mathrm{OCH}_{3}\right), 3.96\left(\mathrm{~d}, J=6.4,2 \mathrm{H}, \mathrm{CH}_{2}\right), 5.78(\mathrm{t}, J=6.4 \mathrm{~Hz}, 1 \mathrm{H}, \mathrm{O}-\mathrm{CH}-$ O), 6.24 (s, 1H, pyrimidine-H), 6.50 (d, $J=7.8 \mathrm{~Hz}, 1 \mathrm{H}, \mathrm{ArH}), 6.99$ (d, $J=7.8 \mathrm{~Hz}, 1 \mathrm{H}, \mathrm{Ar}-\mathrm{H})$. MS [m/z]: $346\left(\mathrm{M}^{+}, 12 \%\right)$. Analysis calcd. for $\mathrm{C}_{17} \mathrm{H}_{22} \mathrm{~N}_{4} \mathrm{O}_{4}$ : C, 58.95; $\mathrm{H}, 6.40 ; \mathrm{N}, 16.17$. Found: C, $58.71 ; \mathrm{H}, 6.36 ; \mathrm{N}, 16.30 \%$.

\section{Synthesis of acetylated triazolopyrimidine N-glycoside derivatives 13 and 14}

Sodium hydride $(0.015 \mathrm{mmol})$ was added portion wise to a stirred solution of compound $\mathbf{1 0}(0.01 \mathrm{~mol})$ in dry DMF $(20 \mathrm{~mL})$ at $0^{\circ} \mathrm{C}$ then stirring was continued for additional $1 \mathrm{~h}$ at room temperature. The sugar 5a, b $(0.01 \mathrm{~mol})$ in dry DMF $(12 \mathrm{~mL})$ has been added slowly followed by stirring for 6-7 $\mathrm{h}$ at $20-25{ }^{\circ} \mathrm{C}$. Ice cold water was added with vigorous stirring for 30 minutes and the resulting precipitate was filtered, dried and crystallized from ethanol to afford $\mathbf{1 3}$ or $\mathbf{1 4}$, respectively. 
2-(Acetoxymethyl)-6-(3-(5-methylfuran-2-yl)-7-oxo-5-propyl$[1,2,4]$ triazolo[4,3-a]pyrimidin-8 $(7 H)$-yl)tetrahydro-2H-pyran3,4,5-triyl triacetate (13)

Yield 76\%; m.p. $153-154^{\circ} \mathrm{C}$; IR (KBr): $1753(\mathrm{C}=\mathrm{O})$, $1657 \mathrm{~cm}^{-1}(\mathrm{C}=\mathrm{O}) .{ }^{1} \mathrm{H}$ NMR $\left(\mathrm{CDCl}_{3}\right) \delta / \mathrm{ppm}: 1.09(\mathrm{t}, J=5.8,3 \mathrm{H}$, $\left.\mathrm{CH}_{3}\right), 1.75\left(\mathrm{~m}, 2 \mathrm{H}, \mathrm{CH}_{2}\right), 1.98,2.03,2.15,2.23\left(4 \mathrm{~s}, 12 \mathrm{H}, 4 \mathrm{CH}_{3}\right)$, $2.28\left(\mathrm{t}, J=5.8,2 \mathrm{H}, \mathrm{CH}_{2}\right), 2.38\left(\mathrm{~s}, 3 \mathrm{H}, \mathrm{CH}_{3}\right), 4.12(\mathrm{dd}, 1 \mathrm{H}, J=10.2$ $\left.\mathrm{Hz}, J=3.5 \mathrm{~Hz}, \mathrm{H}-5^{\prime}\right), 4.17$ (dd, $\left.1 \mathrm{H}, J=3.8,10.2 \mathrm{~Hz}, \mathrm{H}-6^{\prime \prime}\right), 4.29$ $\left(\mathrm{dd}, 1 \mathrm{H}, J=11.3,3.8 \mathrm{~Hz}, \mathrm{H}-6^{\prime}\right), 4.91\left(\mathrm{t}, 1 \mathrm{H}, J=3.2 \mathrm{~Hz}, \mathrm{H}-4^{\prime}\right), 5.18$ $\left(\mathrm{dd}, 1 \mathrm{H}, J=6.6,3.2 \mathrm{~Hz}, \mathrm{H}-3^{\prime}\right), 5.33\left(\mathrm{t}, 1 \mathrm{H}, J=6.6 \mathrm{~Hz}, \mathrm{H}-2^{\prime}\right), 5.88$ (d, 1H, J=9.4 Hz, H-1'), 6.22 (s, 1H, pyrimidine-H), 6.52 (d, $J=$ $7.8 \mathrm{~Hz}, 1 \mathrm{H}, \mathrm{ArH}), 6.98$ (d, $J=7.8 \mathrm{~Hz}, 1 \mathrm{H}, \mathrm{Ar}-\mathrm{H}) .{ }^{13} \mathrm{C} \mathrm{NMR}$

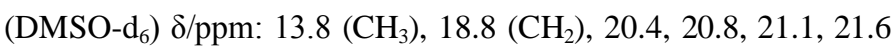
$\left(4 \mathrm{CH}_{3}\right), 38.3\left(\mathrm{CH}_{2}\right), 62.5(\mathrm{C}-6), 68.5(\mathrm{C}-4), 68.8(\mathrm{C}-3), 71.1(\mathrm{C}-2)$, 78.2 (C-5), 91.3 (C-1), 102.6 (pyrimidine C-5), 108.1 (Ar-C), 109.3 (Ar-C), 115.4-156.1 (Ar-C and $2 \mathrm{C}=\mathrm{N}$ ), 162.4, 169.7, 170.1, 170.3, $171.9(5 \mathrm{C}=\mathrm{O})$. Analysis calcd. for $\mathrm{C}_{27} \mathrm{H}_{32} \mathrm{~N}_{4} \mathrm{O}_{11}$ : C, 55.10; H, 5.48; N, 9.52. Found: C, 54.89; H, 5.37; N, 9.41\%.

\section{2-(3-(5-Methylfuran-2-yl)-7-oxo-5-propyl-[1,2,4]triazolo[4,3- a]pyrimidin-8(7H)-yl)tetrahydro-2H-pyran-3,4,5-triyl triacetate (14)}

Yield 78\%; m.p. $158-159^{\circ} \mathrm{C}$; IR (KBr): $1753(\mathrm{C}=\mathrm{O})$, $1670 \mathrm{~cm}^{-1}(\mathrm{C}=\mathrm{O}) .{ }^{1} \mathrm{H}$ NMR $\left(\mathrm{CDCl}_{3}\right) \delta / \mathrm{ppm}: 1.06(\mathrm{t}, J=5.8,3 \mathrm{H}$, $\left.\mathrm{CH}_{3}\right), 1.73\left(\mathrm{~m}, 2 \mathrm{H}, \mathrm{CH}_{2}\right), 1.98,2.03,2.19\left(3 \mathrm{~s}, 9 \mathrm{H}, 3 \mathrm{CH}_{3}\right), 2.27$ (t, $\left.J=5.8,2 \mathrm{H}, \mathrm{CH}_{2}\right), 2.37\left(\mathrm{~s}, 3 \mathrm{H}, \mathrm{CH}_{3}\right), 4.15(\mathrm{dd}, 1 \mathrm{H}, J=2.8,10.2 \mathrm{~Hz}$, H-5") 4.28 (dd, 1H, J= 11.0, $\left.2.8 \mathrm{~Hz}, \mathrm{H}-5^{\prime}\right), 4.90$ (t, 1H, J=3.2 Hz, H-4'), 5.18 (dd, $\left.1 \mathrm{H}, J=6.6,3.2 \mathrm{~Hz}, \mathrm{H}-3^{\prime}\right), 5.34$ (t, $1 \mathrm{H}, J=6.6 \mathrm{~Hz}$, $\mathrm{H}-2^{\prime}$ ), 5.87 (d, 1H, J=9.8 Hz, H-1'), 6.24 (s, 1H, pyrimidine-H), 6.54 (d, $J=7.8 \mathrm{~Hz}, 1 \mathrm{H}, \mathrm{ArH}), 6.99$ (d, $J=7.8 \mathrm{~Hz}, 1 \mathrm{H}, \mathrm{Ar}-\mathrm{H}) .{ }^{13} \mathrm{C}$ NMR (DMSO-d $\left.{ }_{6}\right) \delta /$ ppm: $13.5\left(\mathrm{CH}_{3}\right), 18.5\left(\mathrm{CH}_{2}\right), 20.9,21.1,21.2$ $\left(3 \mathrm{CH}_{3}\right), 39.4\left(\mathrm{CH}_{2}\right), 62.5(\mathrm{C}-5), 67.1(\mathrm{C}-4), 67.9(\mathrm{C}-3), 73.4(\mathrm{C}-2)$, 91.1 (C-1), 102.8 (pyrimidine C-5), 108.4 (Ar-C), 108.6 (Ar-C), 114.7-156.4 (Ar-C and $2 \mathrm{C}=\mathrm{N}), 163.7,170.3,170.4,170.5$ $(4 \mathrm{C}=\mathrm{O})$. Analysis calcd. for $\mathrm{C}_{24} \mathrm{H}_{28} \mathrm{~N}_{4} \mathrm{O}_{9}: \mathrm{C}, 55.81 ; \mathrm{H}, 5.46 ; \mathrm{N}$, 10.85. Found: C, 55.69; H, 5.32; N, 11.05\%.

\section{Synthesis of deacetylated N-glycosides 15 and 16}

The acetylatedglycoside $\mathbf{1 3}$ or $\mathbf{1 4}(5 \mathrm{mmol})$ was added to a saturated methanolic ammonia solution $(15 \mathrm{~mL})$ at $0^{\circ} \mathrm{C}$ was stirring over a period of 20 minutes then the reaction mixture was further stirred at room temperature for $7 \mathrm{~h}$. After completion of the deacetylation process (TLC: petroleum ether/hexane, 2:1), removing the solvent under vacuum gave a yellowish residue. Trituration with cold diethyl ether $(20 \mathrm{~mL})$ with stirring afforded a solid has been filtered and recrystallized using cold ethanol giving compounds 15 and 16, respectively.

\section{3-(5-Methylfuran-2-yl)-5-propyl-8-(3,4,5-trihydroxy-6-} (hydroxymethyl)-tetrahydro-2H-pyran- 2-yl)-[1,2,4]triazolo [4,3-a]pyrimidin-7(8H)-one (15)

Yield 72\%; m.p. $192-193^{\circ} \mathrm{C}$; IR (KBr): 3490-3446 (OH), $1658 \mathrm{~cm}^{-1}(\mathrm{C}=\mathrm{O}) .{ }^{1} \mathrm{H}$ NMR (DMSO- d6) $\delta / \mathrm{ppm}: 1.05(\mathrm{t}, J=5.8$, $\left.3 \mathrm{H}, \mathrm{CH}_{3}\right), 1.72\left(\mathrm{~m}, 2 \mathrm{H}, \mathrm{CH}_{2}\right), 2.27$ (t, $\left.J=5.8,2 \mathrm{H}, \mathrm{CH}_{2}\right), 2.36(\mathrm{~s}$,
$3 \mathrm{H}, \mathrm{CH}_{3}$ ), 3.40-3.48 (m, 2H, H-6', H-6' $), 3.62$ (m, 1H, H-5'), 3.94$4.14\left(\mathrm{~m}, 2 \mathrm{H}, \mathrm{H}-4^{\prime}, 3^{\prime}\right), 4.48\left(\mathrm{~m}, 2 \mathrm{H}, \mathrm{OH}\right.$ and $\left.\mathrm{H}-2^{\prime}\right), 4.85-4.89(\mathrm{~m}$, $1 \mathrm{H}, \mathrm{OH}), 5.14-5.19(\mathrm{~m}, 1 \mathrm{H}, \mathrm{OH}), 5.28(\mathrm{~m}, 1 \mathrm{H}, \mathrm{OH}), 5.81(\mathrm{~d}, 1 \mathrm{H}$, $\left.J=9.2 \mathrm{~Hz}, \mathrm{H}-1^{\prime}\right), 6.23$ (s, 1H, pyrimidine-H), 6.64 (d, $J=7.8 \mathrm{~Hz}$, 1H, ArH), 6.97 (d, $J=7.8 \mathrm{~Hz}, 1 \mathrm{H}, \mathrm{Ar}-\mathrm{H})$. Analysis calcd. for $\mathrm{C}_{19} \mathrm{H}_{24} \mathrm{~N}_{4} \mathrm{O}_{7}$ : C, 54.28; H, 5.75; N, 13.33. Found: C, 54.11; H, $5.59 ; \mathrm{N}, 13.18 \%$.

3-(5-Methylfuran-2-yl)-5-propyl-8-(-3,4,5trihydroxytetrahydro-2H-pyran-2-yl)-[1,2,4]triazolo $[4,3-$ a]pyrimidin-7(8H)-one (16)

Yield 76\%; m.p. $197-198^{\circ} \mathrm{C}$; IR (KBr): 3490-3446 (OH), $1650 \mathrm{~cm}^{-1}(\mathrm{C}=\mathrm{O}) .{ }^{1} \mathrm{H}$ NMR (DMSO- d6) $\delta / \mathrm{ppm}: 1.04(\mathrm{t}, J=5.8$, $\left.3 \mathrm{H}, \mathrm{CH}_{3}\right), 1.72\left(\mathrm{~m}, 2 \mathrm{H}, \mathrm{CH}_{2}\right), 2.26\left(\mathrm{t}, J=5.8,2 \mathrm{H}, \mathrm{CH}_{2}\right), 2.34(\mathrm{~s}$, $3 \mathrm{H}, \mathrm{CH}_{3}$ ), 3.38-3.47 (m, 2H, H-5',H-5'), 3.96-4.15 (t, $1 \mathrm{H}, J=4.8$ $\left.\mathrm{Hz}, \mathrm{H}-4^{\prime}, 3^{\prime}\right), 4.46$ (m, 2H, OH and $\left.\mathrm{H}-2^{\prime}\right), 4.83-4.88$ (m, 1H, OH), $5.22(\mathrm{~m}, 1 \mathrm{H}, \mathrm{OH}), 5.80\left(\mathrm{~d}, 1 \mathrm{H}, J=9.2 \mathrm{~Hz}, \mathrm{H}-1^{\prime}\right), 6.23(\mathrm{~s}, 1 \mathrm{H}$, pyrimidine-H), 6.65 (d, $J=7.8 \mathrm{~Hz}, 1 \mathrm{H}, \mathrm{ArH}), 6.98(\mathrm{~d}, J=7.8 \mathrm{~Hz}$, $1 \mathrm{H}, \mathrm{Ar}-\mathrm{H})$. Analysis calcd. for $\mathrm{C}_{18} \mathrm{H}_{22} \mathrm{~N}_{4} \mathrm{O}_{6}: \mathrm{C}, 55.38 ; \mathrm{H}, 5.68 ; \mathrm{N}$, 14.35. Found: C, 55.17; H, 5.49; N, 14.21\%.

\section{Anticancer Activity \\ Material}

All cell lines were brought from ATCC via Vacsera tissue culture laboratories. All media were purchased from Lonza, Belgium, serum from Gibco, trypsin and MTT from Biobasic Canada.

\section{In vitro antitumor bioassay on human tumor cell lines Cell culture}

MCF7, PC3 and HCT116 cell lines were maintained in DMEM high glucose with l-glutamine, $10 \%$ foetal bovine serum at $37^{\circ} \mathrm{C}$ in $5 \% \quad \mathrm{CO}_{2}$ and $95 \%$ moisture. Cells were sub-cultured employing trypsin versene $0.15 \%$.

Viability test

After nearly $24 \mathrm{~h}$ of cultivating 20000 cells per well (in 96-well plates), when about $60-70 \%$ confluence have attained by cells, alteration of the medium into \% serum-free medium, exhibiting a definitive concentration of compounds of $100 \mu \mathrm{M}$ in triplicates, occurred. Treatment of cells was performed for $72 \mathrm{~h}$. $100 \mu \mathrm{M}$ of Doxorubicin was used as a positive control and serum free medium was used as a negative control.

\section{In vitro antitumor bioassay on human normal cell line Cell culture}

Human retinal pigmented epithelial cell line RPE1 was maintained in DMEM F12 medium, in addition to the same applied conditions and reagents for the cancer cell lines in the present study. In addition the viability test was carried, by seeding 40000 cell lines, as previously mentioned methodology for such latter cell lines.

The cell viability was investigated by applying the MTT (3-(4,5-dimethylthiazol-2-yl)-2,5-diphenyltetrazolium bromide) assay (Mosmann, 1983). 


\section{Applied equation for cytotoxicity}

$$
(1-(\operatorname{av}(\mathrm{x}) /(\operatorname{av}(\mathrm{NC}))) * 100
$$

$\mathrm{X}$ : absorbance of sample well measured at $595 \mathrm{~nm}$ with reference $690 \mathrm{~nm}$,

NC: absorbance of negative control measured at $595 \mathrm{~nm}$ with reference $690 \mathrm{~nm}$

\section{Docking study}

Docking study of the most active antiproliferative compounds 7, 8, 10 and 12 were performed by Molecular Operating Environment (MOE) 2008.10 releases of Chemical Computing Group, Montereal, Canada (http://www.chemcomp. com.). The program operated on an Intel(R) core (TM) i3-32100 CPU@3.10GHz $3.09 \mathrm{GHz}$, processor, 3.41 GB of RAM, Microsoft Windows XP.

Docking was performed against to the active site of the protein molecular surface of CDK2 (PDB ID: 2a4l) in complex with Roscovitine (was downloaded from protein data bank (http://www. rcsb. org/-pdb) (PDB ID: 2a4l) (Stakeret al., 2005).

Prepared of the protein crystal structure for docking was carried out by excluding by water molecules, providing and elimination of polar hydrogen atoms then separation of the active pocket. The active site was believed as the site where Roscovitine (as co-crystalline ligand) may complexe (PDB ID: 2a4l). Roscovitine ligand was re-docked in such pocket to assure the docking methodology is effective in addition that the applied pocket was most convenient for docking emulation of the synthesized products (ligands).

The structure of the selected compounds (ligands) for docking was drawn in ChemDraw Ultra 10.0 (ChemOffice package) and saved. Preparation steps which should precede docking process, involve; a) Visualizing the 3D form of ligands through conversion of the their 2D structure; b) Insertion and excluding polar $\mathrm{H}$ atoms; c) Applying MMFF94x force field to minimize energy till a RMSD (Root-mean-square deviation) of atomic position gradient of $0.01 \mathrm{Kcal} \mathrm{mol}^{-1} \AA$ - 1 was reached and saved as moe (Kaminski et al., 1996).

The docking Algorithm was done by MOE-DOCK default. It uses flexible, rigid technique for posing the molecule inside the cavity. All rotatable bonds of ligands are allowed to undergo free rotation to be placed into the rigid receptor binding site. The docking scores were displayed in negative energy expression; low binding free energy is an indication of better binding affinity (Lensink et al., 2007), and the ligand interactions (hydrogen bonding and hydrophobic interaction) with CDK 2 was determined.

\section{RESULTS AND DISCUSSION}

\section{Chemistry}

The starting 2-hydrazinyl pyrimidine derivative $\mathbf{1}$ was prepared as previously reported (Libermann and Rouaix, 1955). Condensation of alkyl substituted 2-hydrazinyl derivative 1 with 5methylfurfural in presence of acetic acid resulted in the formation of (furylmethylene)hydrazinyl derivative $\mathbf{2}$. The produced (methylene)hydrazinyl compound $\mathbf{2}$ was used as a key compound for the preparation of pyrimidine $N$-substituted acyclic oxygenated alkyl compounds and $\mathrm{N}$-glycosides. It is well known that 1,3disubstituted pyrimidine compounds are synthesized by $N^{3}$ alkylation reactions of $N^{I}$-substituted pyrimidine derivatives (Khalafi-Nezhad et al, 2006; Hovinen, 1997; Ogilvie et al., 1979; Priego et al., 2001) in basic medium. Thus, reaction of the substituted pyrimidine 2 with 2-(2-chloroethoxy)ethan-1-ol and 2chloro-1,1-dimethoxyethane using sodium hydride in $\mathrm{N}, \mathrm{N}$ dimethylformamide afforded their corresponding $N^{3}$-substituted pyrimidine compounds with acyclic oxygenated alkyl chain $\mathbf{3}$ and 4 , respectively.

The IR spectrum of the $N$-substituted pyrimidine derivative $\mathbf{3}$ showed the presence of hydroxyl absorption band. The ${ }^{1} \mathrm{H}$ NMR spectra of the $N$-substituted oxygenated alkyl products $\mathbf{3}$ and $\mathbf{4}$ revealed the presence of the signals assigned for the oxygenated alkyl chain in these products. When the key substituted pyrimidine derivative $\mathbf{2}$ was allowed to react with $\mathrm{O}$ acetylated glycopyranosyl bromide $5 \mathrm{a}, \mathrm{b}$ in presence of sodium hydride at room temperature, the corresponding acetylated $N^{3}$ pyrimidine glycosides $\mathbf{6}$ or $\mathbf{7}$ were obtained in $74-76 \%$ yield. Their ${ }^{1} \mathrm{H}$ NMR spectra possessed the signals which are assigned for the alkyl, aryl and sugar part protons.

The anomeric proton signal appeared as doublet with coupling constants 10.2 and $9.5 \mathrm{~Hz}$ indicating that the afforded glycosides in the $\beta$-conformation. Deacetylation of the latter glycosides was performed effectively by means of saturated methanolic ammonia solution at room temperature to give the congruent glycosides with unprotected hydroxyls $\mathbf{8}$ and $\mathbf{9}$, respectively (scheme 1).

Their IR spectra showed the sugar-hydroxyl absorption bands and the disappearance of the acetyl-methyl signals in the corresponding NMR data. On the other hand, compound $\mathbf{2}$ was used also for the preparation of a condensed pyrimidine derivative having a free $\mathrm{NH}$ in the pyrimidine nucleus. The reaction was performed by means of bromine in methanol to result in the $[1,2,4]$ triazolo[4,3-a]pyrimidine derivative 10. The ${ }^{1} \mathrm{H}$ NMR spectrum indicated the absence of proton of the azomethine $(\mathrm{N}=\mathrm{CH})$ group and displayed one $\mathrm{NH}$ signal. Its ${ }^{13} \mathrm{C}$ NMR showed the disappearance of the $C \mathrm{H}=\mathrm{N}$ and presence of the $C-3$ ay higher chemical shift. Formation of the triazolopyrimidine with this mode of cyclization was also confirmed by formation of cyclized product 12 by reaction of substituted pyrimidine 4 with bromine in acetic acid which is the same product of alkylation of triazolopyrimidine $\mathbf{1 0}$ with 2-chloro-1,1-dimethoxyethane. These results are in accordance with previously reported results for similar triazolpyrimine syntheses (Shaban et al., 1995; Turk et al., 1998) and also with their mode of preparation. The prepared triazolopyrimidine $\mathbf{1 0}$ was also used, like the substituted pyrimidine $\mathbf{3}$, to prepare another $\mathrm{N}$-substituted triazolopyrimidine derivative of the triazolopyrimidne system by reaction with oxygenated hydroxyl alkyl; namely 2-(2-chloroethoxy)ethan-1-ol and gave the $N$-substituted derivative 11, in $77 \%$ yield. 
Glycosylation of the key triazolopyrimidine $\mathbf{1 0}$ with the same $\alpha$-glycosyl bromides used previously lead to the formation of the $N^{3}$-triazolopyrimidine glycosides 13 and 14, respectively. The ${ }^{1} \mathrm{H}$ NMR spectra showed, in addition to signals of alkyl and aryl protons, the sugar moiety signals appeared at $4.12-5.88 \mathrm{ppm}$. The coupling constants of the $H-1$ in the glycosyl moiety 9.4 and 9.8 $\mathrm{Hz}$ indicated $\beta$-glycosidic linkage naturein glycosides $\mathbf{1 3}$ and $\mathbf{1 4}$. Preparation of the free hydroxyl glycoside derivatives $\mathbf{1 5}$ and $\mathbf{1 6}$ was performed by the deacetylation reaction of the acetylated $N$ glycosides 13 and $\mathbf{1 4}$, respectively by means of ammonia solution in dry methanol at room temperature. The IR spectra of the resulting deprotected glycosides showed the hydroxyl bands in addition to the disappearance of the acetyl-carbonyl functions. Their ${ }^{1} \mathrm{H}$ NMR spectra indicated the presence of the hydroxyl and sugar protons signals in addition to the disappearance of the methyl of the acetyl groups.

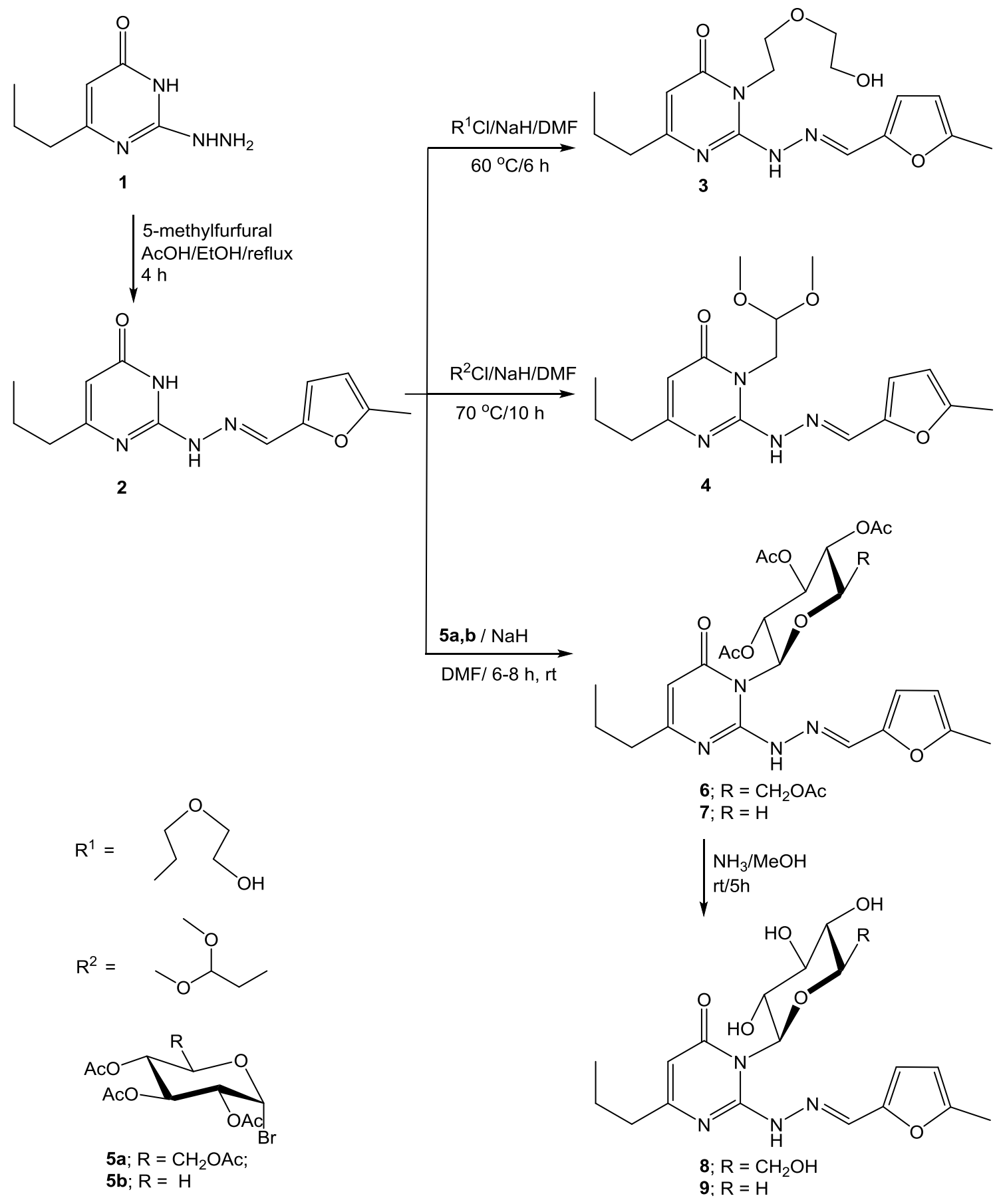

Scheme 1 Synthesis of pyrimidine glycosides and acyclic analogs. 


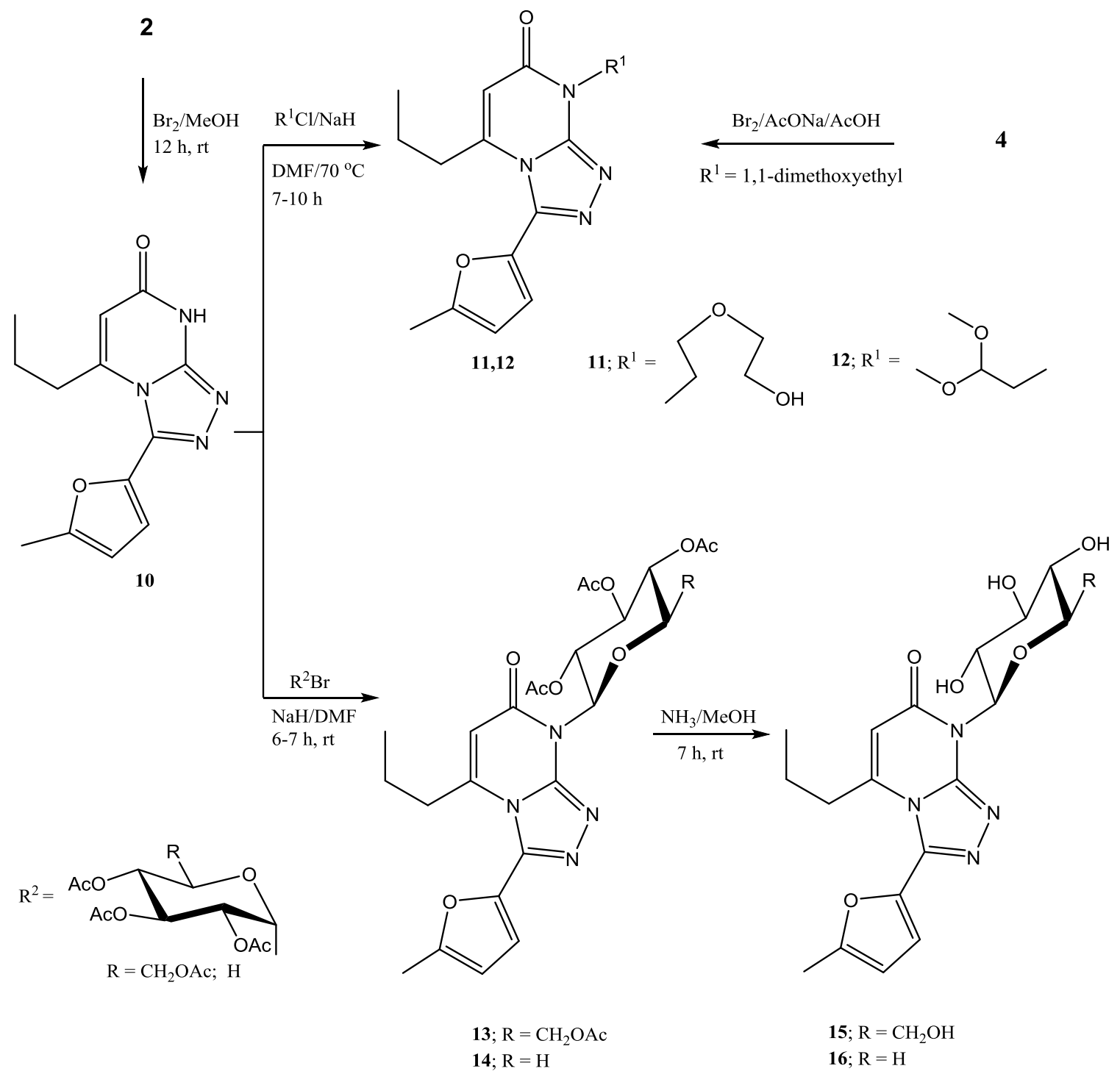

Scheme 2: Synthesis of triazolopyrimidine glycosides.

\section{Anticancer Activity}

The anticancer activity of the synthesized compounds was studied on human prostatic adenocarcinoma (PC3), human colorectal carcinoma (HCT116) and human breast adenocarcinoma (MCF7) cell lines in addition to their effect on human normal retinal pigmented epithelial cell line (RPE1) using the MTT assay (Mosmann, 1983; El-Ansary et al., 2015). The results were shown in table 1 and fig. 2, expressed as the cytotoxic effect of the samples at $100 \mu \mathrm{M}$ and reflected the effect of substitution at the $N$ 3 position in the pyrimidine and triazolopyrimidine ring systems. The inhibition activity of the $N^{3}$-unsubstituted analogs of the latter heterocycles were also shown in table 1.

By reviewing the results, it was revealed that the $N$ substituted pyrimidine derivatives $\mathbf{7}$ and $\mathbf{8}$ in addition to triazolopyrimidine compounds $\mathbf{1 0}$ and $\mathbf{1 2}$ showed moderate activity compound $\mathbf{1 0}$ showed activity on the three tumor cell lines with inhibition 48, 40 and 46\% on PC3, HCT116 and MCF7, respectively. Interestingly, it was found that such compound gave $20 \%$ less toxicity on the normal cell line and so it is of some specificity on cancer cell lines. Compound $\mathbf{8}$ gave more $62 \%$ on PC3 and 55\% against HCT116. Compounds 7 and 12 showed 55 and $51 \%$ inhibition, respectively on human prostatic adenocarcinoma (PC3). As a result, although $\mathbf{1 0}$ is of lower effect than compounds 7, 8 and $\mathbf{1 2}$ on cancer cells under test, it is more of interest as it is selective on cancer cells rather than normal cells.

In correlation of the structure of active compounds with the obtained results, it is shown that attachment of glycosyl moiety to the pyrimidine ring system resulted in increased inhibition activity. This was observed for the obtained inhibition results on the three cell lines as the activity was elevated in the synthesized glycosides $\mathbf{7}$ and $\mathbf{8}$ in comparison with the free pyrimidine $\mathbf{3}$. In addition the condensed attachment of 1,2,4-triazole nucleus to the 
pyrimidine ring system afforded higher inhibition activity which is obvious from the higher activity of the triazolopyrimidine $\mathbf{1 0}$ derivative than its pyrimidine precursor. Furthermore, the substitution at $N$-1position in triazolpyrimidine nucleus with acyclic oxygenated alkyl, as triazolopyrimidine acyclic nucleoside analog, lead to raised inhibition activity on PC3 compared to the terminal free hydroxyl substituent. Moreover, it was concluded that the substituted pyrimidine glycosides were found to be more active than their derived triazolpyrimidine glucoside or xyloside. These results could be basis of further studies for design and synthesis of more modified pyrimidine nucleoside analogs.

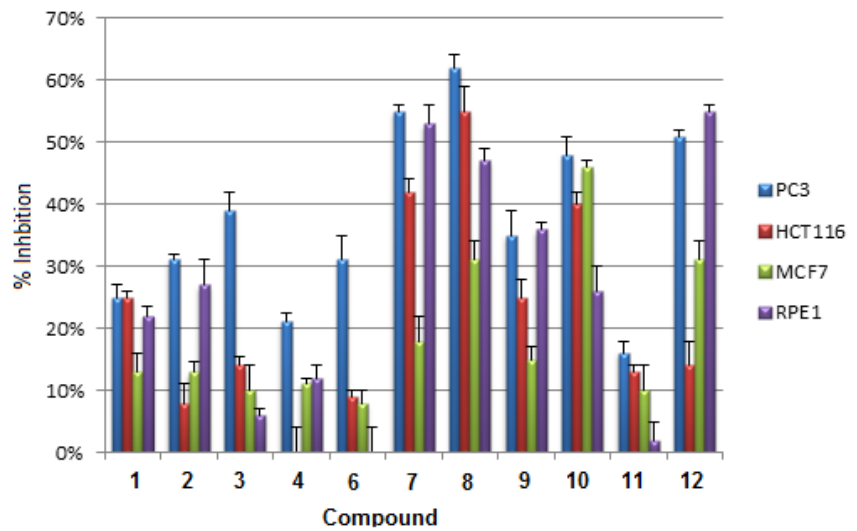

Fig. 2: The cytotoxic effect of the samples at $100 \mu \mathrm{M}$ on human prostatic adenocarcinoma (PC3), human colorectal carcinoma (HCT116) and human breast adenocarcinoma (MCF7) cell lines.

Table 1: Cytotoxic effect compounds at $100 \mu \mathrm{M}$ on PC3, HCT116, MCF7 cell lines and human normal retinal pigmented epithelial cell line (RPE1).

\begin{tabular}{ccccc}
\hline Compound & PC3 & HCT116 & MCF7 & RPE1 \\
\hline $\mathbf{2}$ & $31 \%$ & $8 \%$ & $13 \%$ & $27 \%$ \\
$\mathbf{7}$ & $55 \%$ & $42 \%$ & $18 \%$ & $53 \%$ \\
$\mathbf{8}$ & $62 \%$ & $55 \%$ & $31 \%$ & $47 \%$ \\
$\mathbf{1 0}$ & $48 \%$ & $40 \%$ & $46 \%$ & $26 \%$ \\
$\mathbf{1 2}$ & $51 \%$ & $14 \%$ & $31 \%$ & $55 \%$ \\
\hline
\end{tabular}

\section{Molecular docking study}

Docking studies are coveted so as to comprehend the mechanisms of actions of drugs, modes of interactions with targets, and to integrate any experimental guide announced. These forms are needed to get a suitable and more accurate form of biologically active molecules at the atomic level and thus, provide new willfulness which might be applied to design novel therapeutic agents. Docking process was performed for the target compounds intocyclin-dependent kinase 2 (CDK2) using MOE 2008.10 program.

From the obtained results (table 3, fig. 4-7) it was shown that, the studied compounds exhibited good fitting ability inside the binding site of the protein molecular surface with minimum binding energy ranged from -13.432 to $-22.760 \mathrm{~kJ} \mathrm{~mol}^{-1}$ in comparison to the co-crystallized ligand. Co-crystallized ligand Roscovitine exhibited binding energy of $-23.54 \mathrm{kJmol}^{-1}$ and it showed van der Waals interaction with arene-cationand between Lys 89 (Fig 3).

Compound 7 showed binding energy of-21.569 kJ mol${ }^{-1}$ and formed one hydrogen bond with the oxygen atom of the carbonyl group moiety as it acts as a hydrogen bond acceptor with the side chain of Lys 89 in distance $2.53 \AA$ with strength of $19 \%$ (Fig 4). Substitution at $\mathrm{C}$-2 in the pyrimidine ring with substituted amino group linked to oxygenated moiety provides some similarity to the structure of Roscovitine. The docking conformation of compound $\mathbf{8}$ in the active site of the protein revealed good interactions with the active site residues of this protein. Compound 8 formed hydrogen bond interaction between hydrogen of hydroxyl group moiety with the side chain of Glu 12 residue (2.60 $\AA$ ) with a strength of $33 \%$. Furthermore, it showed van der Waals interaction with Lys 89 (Figure 5).

The absence of the hydroxyls responsible for the $\mathrm{H}$ bonds accounts for the binding energy of this compound compared to its acetylated form. Meanwhile, docking study of compound $\mathbf{1 0}$ showed hydrogen bond interaction between oxygen atom of the carbonyl group moiety and the side chain of Lys89 residue (2.51 $\AA$ ) with strength of $35 \%$ (Figure 6). Docking studies of compound 12 into the active site of the enzyme showed two hydrogen bonds one between oxygen of carbonyl group moiety and Lys89 residue $(2.32 \AA)$ and the other between oxygen of the methoxy group and the same protein residue $(2.71 \AA)$ with strength of $15 \%$ and $62 \%$ respectively (Figure 7).

The conformation of the acyclic substituent at $N-3$ in the pyrimidine ring with acyclic oxygenated alkyl having two symmetrical methoxy groups might be with effect allowing for such binding mode.

Table 2: $\mathrm{IC}_{50}$ values for compounds which showed more than $40 \%$ inhibition.

\begin{tabular}{|c|c|c|c|c|c|c|c|c|}
\hline \multirow{2}{*}{ Compd. } & \multicolumn{2}{|c|}{ PC3 } & \multicolumn{2}{|c|}{ HCT116 } & \multicolumn{2}{|c|}{ MCF7 } & \multicolumn{2}{|c|}{ RPE1 } \\
\hline & $\mathrm{IC}_{50}$ & $\mathrm{IC}_{90}$ & $\mathrm{IC}_{50}$ & $\mathrm{IC}_{90}$ & $\mathrm{IC}_{50}$ & $I_{C_{90}}$ & $\mathrm{IC}_{50}$ & $I_{90}$ \\
\hline 7 & $75 \pm 4$ & $149 \pm 11$ & $93 \pm 8$ & $155 \pm 13$ & - & - & $66 \pm 6$ & $146.9 \pm 10$ \\
\hline 8 & $70 \pm 9$ & $123 \pm 12$ & $95 \pm 16$ & $144 \pm 20$ & - & - & $93 \pm 9$ & $172 \pm 21$ \\
\hline 10 & $98 \pm 9$ & $138 \pm 14$ & $101 \pm 7$ & $169 \pm 12$ & $103 \pm 9$ & $160 \pm 13$ & - & - \\
\hline 12 & $87 \pm 7$ & $177 \pm 18$ & - & - & & & $148 \pm 18$ & $245 \pm 20$ \\
\hline DOX. & $6.8 \pm 1.2$ & $13.8 \pm 0.8$ & $2.2 \pm 3.1$ & $5.2 \pm 1.9$ & $12.8 \pm 1$ & $51.7 \pm 0.7$ & - & - \\
\hline
\end{tabular}

Table 3: Binding energies and distance of compounds 7, 8, 10 and 12.

\begin{tabular}{|c|c|c|c|c|}
\hline Compounds & Binding $\mathbf{E}\left(\mathrm{KJ} \mathrm{mol}^{-1}\right)$ & Main atoms & Residue & Distance $\mathrm{A}^{\circ}$ \\
\hline 7 & -21.569 & oxygen atom of the carbonyl group moiety & Lys 89 & 2.53 \\
\hline 8 & -19.9115 & hydrogen of hydroxyl group moiety & Glu 12 & 2.60 \\
\hline 10 & -22.760 & oxygen atom of the carbonyl group moiety & Lys 89 & 2.51 \\
\hline 12 & -13.432 & $\begin{array}{c}\text { oxygen of carbonyl group moiety } \\
\text { oxygen of the methoxy group }\end{array}$ & $\begin{array}{l}\text { Lys89 } \\
\text { Lys89 }\end{array}$ & $\begin{array}{l}2.32 \\
2.71 \\
\end{array}$ \\
\hline
\end{tabular}




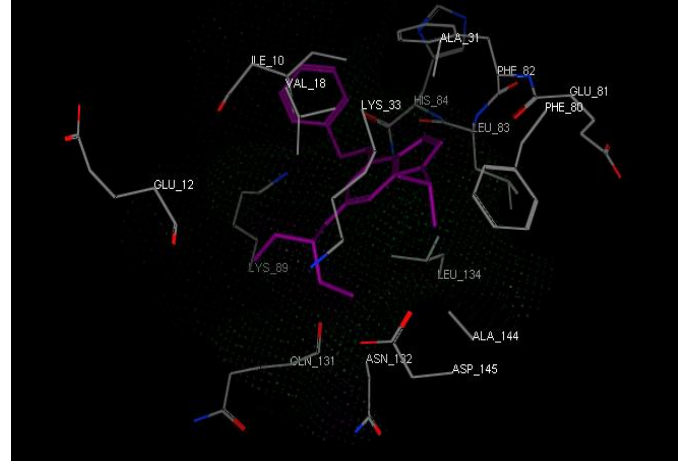

Fig 3. Docking of compound Roscovitineinto the active site of CDK2.

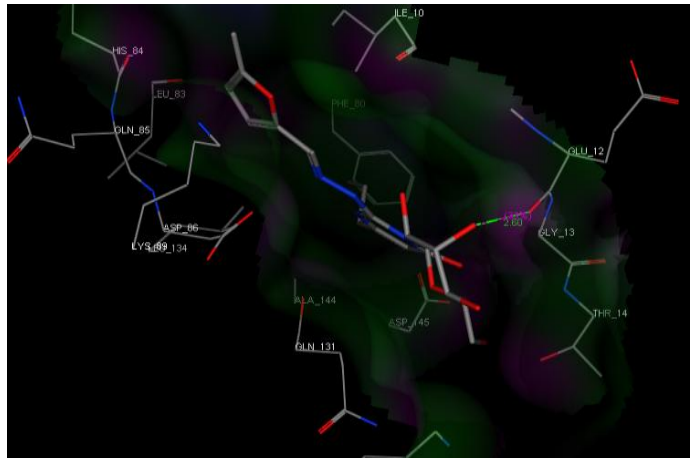

Fig 5. Docking of compound 8into the active site of CDK2.

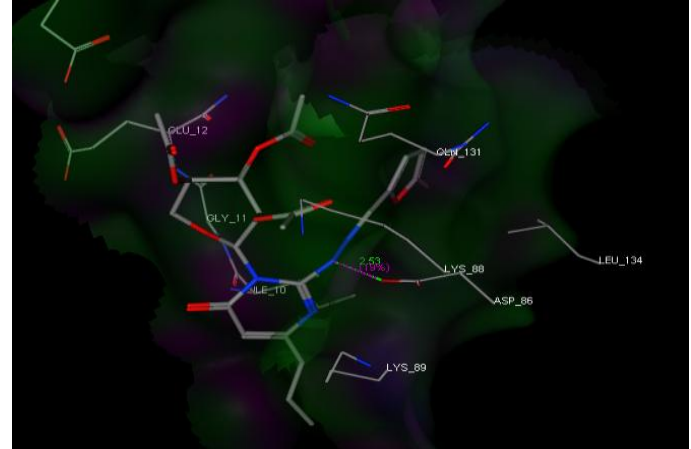

Fig 4. Docking of compound 7into the active site of CDK2.

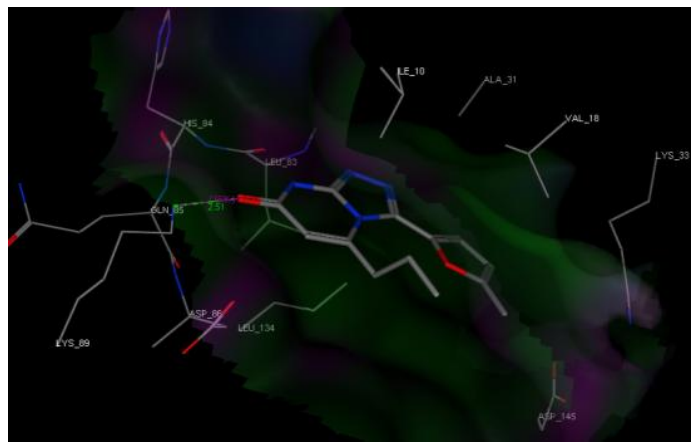

Fig 6. Docking of compound 10into the active site of CDK2.

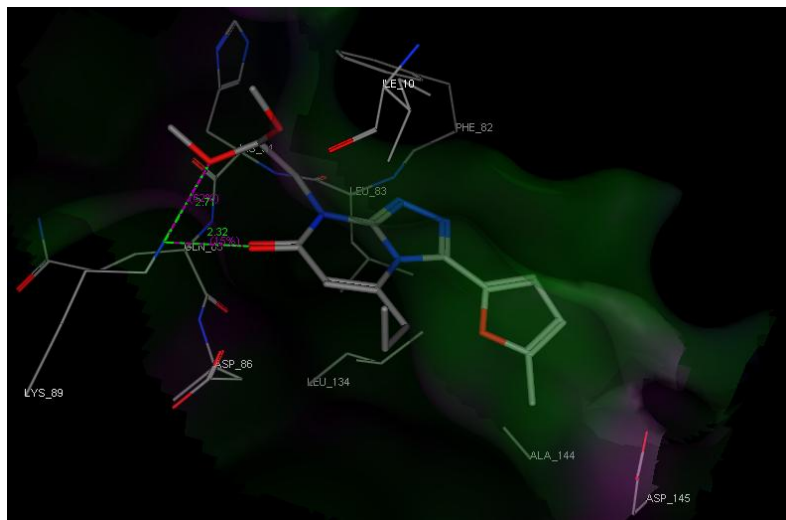

Fig 7. Docking of compound 12into the active site of CDK2.

\section{CONCLUSION}

New substituted pyrimidine and triazolopyrimidine glycosides as well as their acyclic analogs were synthesized and studied for their anticancer activity. Some of the synthesized compounds showed good activity and triazolopyrimidine derivative have been shown to be selective to cancer cells.

\section{Financial support and sponsorship: Nil.}

Conflict of Interests: There are no conflicts of interest.

\section{REFERENCES}

Al-Harbi NO, Bahashwan SA, Fayed AA, MS, Amr A, G. Antiparkinsonism, hypoglycemic and anti-microbial activities of new poly fused ring heterocyclic candidates. Int J Biol Macromol 2013; 57: $165-73$.
Al-Mohizea AM, Al-Omar MA, Abdalla MM, Amr AG. alphareductase inhibitors, antiviral and anti-tumor activities of some steroidal cyanopyridinone derivatives. Int J Biol Macromol 2012; 50: 171-79.

Aminin DL, Menchinskaya ES, Pislyagin EA, Silchenko AS, Avilov SA, Kalinin VI. Sea Cucumber Triterpene Glycosides as Anticancer Agents. Studies in Natural Products Chemistry. 2016; 49: 55105

Amr AE, Mohamed AM, Mohamed SF, Abdel-Hafez NA, Hammam AG. Anticancer activity of some newly synthesized pyridine, pyrane, and pyrimidine derivatives. Bioorg Med Chem 2006; 4: 5481-5488.

Butler T, Maravent S, Boisselle J, Valdes J, Fellner C. A review of 2014 cancer drug approvals, with a look at 2015 and beyond. Pharmacol Ther 2015; 40: 191-205.

Champoux JJ DNA topoisomerases: structure, function, and mechanism Annu. Rev Biochem 2001; 70: 369-13.

Cieplik J. Pyrimidines as the cytostatic drugs. Acta Pol Pharm 1992; 49: 51-57.

El-Ansary AKE, Mohamed NA, Mohamed KO, Abd-Elfattah HMW, El-Manawaty MA. Simple and Convenient Synthesis of Novel 
Thiopyrimidine Derivatives as Anticancer Agents. Res J Pharm Biol Chem Sci 2015; 6: 1745-52.

El-Sayed WA, Abbas HS, Abdel Magid RE, Magdziarz T. Synthesis, Antimicrobial Activity and Docking Studies of New 3(Pyrimidin-4-yl)- $1 H$-indole Derivatives and Their Derived $\mathrm{N}$ - and Sglycoside Analogs. Med Chem Res 2016; 25: 339-55.

El-Sayed WA, Rashad, AE, Awad SM, Ali MM. Synthesis and in vitro Antitumor Activity of Some New Substituted Thiopyrimidines Through Radical Balance Regulation. Nucleosides, Nucleotides and Nucleic Acids. 2009; 28: 261-74

Gui M, Shi DK, Huang M, Zhao Y, Sun QM, Zhang J, Chen Q, Feng JM, Liu CH, Li M, Li YX, Geng M.Y, Ding J. a novel glycosylated diphyllin derivative, exhibits potent anticancer activity by targeting topoisomerase II $\alpha$. Invest. New Drugs. 2011; 29: 800-10.

Hafez HN, El-Gazzar ABA. Synthesis and antitumor activity of substituted triazolo[4,3-a]pyrimidin-6-sulfonamide with an incorporated thiazolidinone moiety. Bioorg Med Chem Lett 2009; 19: 4143-47.

Hannah DR, Sherer EC, Davies RV, Titman RB, Laughton CA, Stevens MFG. Structural studies on bioactive compounds. Part 29: Palladium catalysedarylations and alkynylations of sterically hindered immunomodulatory 2-amino-5-halo-4,6-(disubstituted)pyrimidines. Bioorg Med Chem 2000; 8: 739-50

Hossan AS, Abu-Melha HM, Al-Omar MA,AmrAel G. Synthesis and antimicrobial activity of some new pyrimidinone and oxazinone derivatives fused with thiophene rings using 2-chloro-6-ethoxy4-acetylpyridine as startingmaterial. Molecules 2012; 17: 13642-55.

Hovinen J. Selective $\mathrm{N}^{3}$ - and 5'-O-Alkylation of 2',3'-OIsopropylideneuridine with Methyl Iodide. Helv Chim Acta1997; 80: 85155.

Jordheim, LP, Durantel D, Zoulim F, Dumontet C. Advances in the development of nucleoside and nucleotide analogues for cancer and viral diseases. Nat Rev Drug Disc 2013; 12: 447-64.

Kaminski G, Jorgensen WL. Performance of the AMBER94, MMFF94 and OPLS-AA force fields for modeling organic liquids. J Phys Chem 1996; 100: 18010-3.

Khalafi-Nezhad A, Zare A, Parhami A, Soltani Rad MN. Practical synthesis of some novel unsymmetrical 1,3-dialkylpyrimidine derivatives at room temperature. Arkivoc 2006; xii: 161-172.

Khan SI, Grinstaff MW. A facile and convenient solid-phase procedure for synthesizing nucleoside hydroxamic acids. Tetrahedr Lett 1998; 39: 8031-34.

Lensink MF, Méndez R, Wodak SJ. Docking and scoring protein complexes: CAPRI 3rd Edition. Proteins 2007; 69: 704-18.

Libermann D, Rouaix C. Sur Les Hydrazino-pyrimidines. C R Hebd Seances Acad Sci 1955; 240: 984-86.

Malagu K, Duggan H, Menear K, Hummersone M, Gomez S, Bailey C, Edwards P, Drzewiecki J, Leroux F, Quesada MJ, Hermann G, Maine S, Molyneaux C, Le Gall A, Pullen J, Hickson I, Smith L, Maguire S, Martin N, Smith G, Pass M. The discovery and optimization of pyrido[2,3- $d]$ pyrimidine-2,4-diamines as potent and selective inhibitors of mTOR kinase. Bioorg Med Chem Lett 2009; 19: 5950-53.

McGuian C, Barucki H, Blewett S, Carangio A, Erichsen JT, Andrei G, Snoeck R, De Clercq E, Balzarini J. Highly Potent and Selective Inhibition of Varicella-Zoster Virus by Bicyclic Furopyrimidine Nucleosides Bearing an Aryl Side Chain. J. Med. Chem. 2000; 43: 4993-97

Mohamed AM, Al-Qallawi HRM, El-Sayed WA, Arafa WAA, Alhumaimus MS, Hassan AK Anticancer Activity of Newly Synthesized Triazolopyrimidine Derivatives and Their Nucleoside Analogs. Acta Polon Pharm Drug Res. 2015; 72: 307-18.

Mohamed AM, El-Sayed WA, Alsharari MA, Al-Qalawi HR, Germoush MO. Anticancer activities of some newly synthesized pyrazole and pyrimidine derivatives. Arch. Pharm. Res. 2013; 36: 1055-65.

Mosmann T. Use of MTT colorimetric assay to measure cell activation. J. Immunol. Methods. 1983; 65: 55-63.

Ogilvie KK, Beaucage SL, Gillen MF, Entwistle D, Quilliam M. Fluoride ion catalyzed alkylation of purines, pyrimidines, nucleosides and nucleotides using alkyl halides. Nucleic Acids Res 1979; 6: 1695-08.

Palmer BD, Smaill JB, Rewcastle GW, Dobrusin EM, Kraker A, Moore CW, Steinkampf RW, Denny WA. Structure-activity relationships for 2-anilino-6-phenylpyrido[2,3- $d]$ pyrimidin-7(8H)-ones as inhibitors of the cellular checkpoint kinase Wee1. Bioorg Med Chem Lett 2005; 15:1931-35

Pogorelcnik B, Brvar M, Zegura B, Filipic M, Solmajer T, Perdih A. Discovery of mono- and disubstituted $1 \mathrm{H}_{-}$ pyrazolo[3,4]pyrimidines and $9 \mathrm{H}$-purines as catalytic inhibitors of human DNA topoisomerase IIalpha. Chem Med Chem 2015; 10: 345-59.

Pommier Y, Leo E, Zhang HL, Marchand C. DNA Topoisomerases and Their Poisoning by anticancer and antibacterial drugs. Chem Biol 2010; 17: 421-33.

Priego E.-M, Camarasa M.-J, Pérez-Pérez M.-J. Efficient Synthesis of $N$-3-Substituted 6-Aminouracil Derivatives via $N^{6}$ [(Dimethylamino)methylene] Protection. Synthesis 2001; 478-82.

Shaban MAE,Taha MAM, Nasr AZ, Morgan AEA. Synthesis of acyclo $C$-nucleosides: $\quad 3$-(alditol-1-yl)-5-methyl-7-oxo-1,2,4-triazolo[4,3a]pyrimidines. Pharmazie 1995; 50: 784-8.

Shawali AS, Sherif SM, Darwish MAA, El-merzabani MM. Synthesis and Antitumor Screening of New 1,7-Diphenyl-3-(1,3disubstituted-1H-pyrazole-4-carbonyl)-[1,2,4]triazolo[4,3-a]pyrimidin5(1H)-ones. Arch Pharm Res 2010; 33: 55-60.

Shi DK, Zhang W, Ding N, Li M, Li YX. Design, synthesis and biological evaluation of novel glycosylated diphyllin derivatives as topoisomerase II inhibitors Eur J Med Chem. 2012; 47: 424-31.

Srinivasan S, McGuian C, Andrei G, Snoeck R, De Clercq E, Balzarini J. Bicyclic nucleoside inhibitors of Varicella-Zoster virus (VZV): the effect of terminal unsaturation in the side chain Bioorg Med Chem Lett 2001; 11: 391-3

Staker BL,Feese MD, Cushman M, Pommier Y,Zembower D, Stewart L, et al. Structure of three classes of anticancer agents bound to the human topoisomerase I-DNA covalent complex. J Med Chem 2005; 48: $2336-45$.

Toogood PL, Harvey PJ, Repine JT, Sheehan DJ, Vander Wel SN, Zhou H, Keller PR, McNamara DJ, Sherry D,Zhu T, Brodfuehrer J, Choi C, Barvian MR, Fry DW. Discovery of a Potent and Selective Inhibitor of Cyclin-Dependent Kinase. J Med Chem 2005; 48: 2388-96.

Turk C, Sevete J, Golobic A,Golic L, Stanovnic B. Synthesis of 4- and 5-(s-Ttriazolo[4,3-b]pyridazinyl-3]-substituted cyclic polyols. J Het Chem 1998; 35: 513-18.

Vander Wel SN, Harvey, PJ, McNamara DJ, Repine JT, Keller PR, QuinJ, Booth RJ, Elliott WL, Dobrusin E.M, Fry DW, Toogood PL.Pyrido[2,3-d]pyrimidin-7-ones as Specific Inhibitors of CyclinDependent Kinase. J Med Chem 2005; 48: 2371-78.

Wannberg J, Dallinger D, Kappe CO, Larhed M. Microwaveenhanced and metal-catalyzed functionalizations of the 4-aryldihydropyrimidone template. J Comb Chem 2005; 7: 574-83.

Yoo SJ, Kim HO, Lim Y, Kim J, Jeong LS. Synthesis of novel $(2 \mathrm{R}, 4 \mathrm{R})$ - and $(2 \mathrm{~S}, 4 \mathrm{~S})$-isodideoxy nucleosides with exocyclic methylene as potential antiviral agents. Bioorg Med Chem 2002; 10: 215-26.

Zhao XL, ZhaoYF, Guo SC, Song HS, Wang D, Gong P. Synthesis and Anti-tumor Activities of Novel [1,2,4]triazolo[1,5a]pyrimidines. Molecules 2007; 12: 1136-46.

Zhao Y, Lu YP, Zhu L. Synthesis of Per-acetyl d-fucopyranosyl Bromide and Its Use in Preparation of Diphyllin d-fucopyranosyl Glycoside. J Carbohydr Chem 2008; 27: 113-19.

Zhao Y, Ni C, Zhang Y, Zhu L. Synthesis and Bioevaluation of Diphyllin Glycosides as Novel Anticancer Agents. Arch Pharm Chem Life Sci. 2012; 345: 622-8

Zhao Y, Li YX. First Synthesis of Bioactive Diphyllin Glycosides Isolated from Justiciapatentiflor Hemsl. Chin J Chem 2007; 25: 679-82.

How to cite this article:

El-Sayed WA, Mohamed AM, Khalaf HS, EL-Kady DS, AlManawaty M. Synthesis and Anticancer Activity of New Substituted Pyrimidine and Triazolopyrimidine Glycosides. J App Pharm Sci, 2017; 7 (09): 001-011. 